\title{
Weight-to-height ratio and body roundness index are superior indicators to assess cardio-metabolic risks in Chinese children and adolescents: compared with body mass index and a body shape index
}

\author{
Rui Chen ${ }^{1}$, Lang Ji ${ }^{2}$, Yitong Chen ${ }^{3}$, Linghui Meng ${ }^{1}$ \\ ${ }^{1}$ Center for Evidence-Based Medicine, Capital Institute of Pediatrics, Beijing, China; ${ }^{2}$ Central Laboratory, Beijing Luhe Hospital, Capital Medical \\ University, Beijing, China; ${ }^{3}$ Infectious Diseases Control and Prevention Division, Daxing Center for Disease Control and Prevention, Beijing, China \\ Contributions: (I) Conception and design: R Chen, L Meng; (II) Administrative support: L Meng; (III) Provision of study materials or patients: Y \\ Chen, L Ji, L Meng; (IV) Collection and assembly of data: Y Chen, L Ji, R Chen; (V) Data analysis and interpretation: R Chen; (VI) Manuscript \\ writing: All authors; (VII) Final approval of manuscript: All authors. \\ Correspondence to: Prof. Linghui Meng. Center for Evidence-Based Medicine, Capital Institute of Pediatrics, Beijing 100020, China. \\ Email: linghui61@163.com.
}

Objective: To assess the relationship between five anthropometric indicators, which includes body mass index (BMI), weight-to-height ratio (WHtR), a body shape index (ABSI), ABSI-adolescents, and body roundness index (BRI) in Chinese children and adolescents, and select which could better predict cardiometabolic risk factors (CMRFs).

Methods: Cross-sectional study with 1,587 participants aged 3 to 17 years. Five anthropometric indicators were calculated according to weight, height and waist circumference (WC). Anthropometric measurements and laboratory indicators were used to diagnose CMRFs, which included hypertension, dyslipidemia, impaired fasting glucose and abdominal obesity. Partial correlation analysis was used to evaluate the relationship among anthropometric indicators, area under the receiver operating characteristic curve (AUCs) were used to compare the predict ability of each anthropometric indicators, the cut-off value, sensitivity, specificity and Youden Index of each indicator were calculated.

Results: In 3-6 years old children, ABSI-adolescent positively correlated with $\mathrm{WC}(\mathrm{r}=0.727, \mathrm{P}<0.001)$, BMI $(r=0.218, P<0.001)$ and WHtR $(r=0.752, \mathrm{P}<0.001)$, and in $7-17$ years old participants, the correlation coefficients increased to $0.842,0.563$ and $0.850(\mathrm{P}<0.001)$, respectively. BRI were strong correlated with BMI, WHtR and ABSI-adolescents in both age group $(\mathrm{P}<0.001)$. In 3-6 years group, the ROC analysis showed that BMI and ABSI were significantly better in identifying hypertension in both genders, WHtR and BRI were significantly better in identifying abdominal obesity in girls, but all of them were failed in identifying dyslipidemia and hyperglycemia. In 7-17 years group, WHtR and BRI were significantly better in identifying hypertension, dyslipidemia, abdominal obesity in both genders, BMI and ABSI performed better in identifying hyperglycemia in girls.

Conclusions: In Chinese children aged 3-6 years, there is no indicator performed best in all the CMRFs, in 7-17 years old teenagers, WHtR and BRI can be recommended to identify hypertension, dyslipidemia, abdominal obesity and clustered CMRFs in both genders. However, ABSI showed weak discriminative power.

Keywords: Anthropometric indicators; cardiometabolic risk factors; children and adolescents; body roundness index (BRI); a body shape index (ABSI)

Submitted Sep 30, 2021. Accepted for publication Jan 18, 2022.

doi: $10.21037 / \mathrm{tp}-21-479$

View this article at: https://dx.doi.org/10.21037/tp-21-479 


\section{Introduction}

Obesity as a well-known cardiometabolic diseases risk factor, such as hypertension (1), dyslipidemia (2) and diabetes (3). With the prevalence of obesity in children and adolescents, obese-related cardiometabolic diseases are increasing globally (4). Obesity in childhood is associated with increased cardiometabolic risks (4-6). Body mass index (BMI) as the most commonly used indicator to assess obesity (7), still have limitations because of cannot provide the information about fat distribution and cannot distinguish fat accumulation from muscle (8). Waist circumference (WC) can be used to evaluate abdominal obesity; however, WC is highly related to BMI, therefore, it's difficult to consider the two as independent risk factors (9). Furthermore, weight-to-height ratio (WHtR) was considered as a superior and practical screen tool of detecting cardio-metabolic risk factors (CMRFs) in pediatric population $(10,11)$, and $W H t R \geq 0.5$ was suggested as an increased risk for individuals (12).

Two new anthropometric indicators, a body shape index (ABSI) and body roundness index (BRI) were originally developed by Krakauer et al. (13) and Thomas et al. (14), respectively. The authors have claimed that ABSI can be used as a complementary to BMI to provide additional risks caused by high WC, and appears to be a significant risk factor for premature death, and Thomas et al. claimed BRI's predictive ability for body fat percentage (\%BF) and visceral adipose tissue percentage (\%VAT) is slighter higher than BMI, WC and hip circumference. However, there are few studies exploring the ability of ABSI and BRI to predict CMRFs, research conclusions conflict in adults, (15-19) as well as in children and adolescents (20-22). Considering the difference between adolescents and adults, Xu et al. (20) used the data of 562 adolescents aged 10-17 years in the 2009 CHNS, updated the scaling exponents in ABSI, called ABSI-adolescents. Taking these perspectives into account, the aims of present study are: (I) assess the relationship between five anthropometric indices (BMI, WHtR, ABSI, ABSI-adolescents, BRI); (II) compare the capacity of five anthropometric indices (BMI, WHtR, ABSI, ABSIadolescents, BRI) to identify single and clustered CMRFs in children and adolescents. Compared with previous studies, we not only included children aged 3 to 6 years, but also added two new anthropometric indicators (ABSI and BRI) into the comparation, which have not been evaluated between 3 to 17 years old children and adolescents. We present the following article in accordance with the STARD reporting checklist (available at https://tp.amegroups.com/ article/view/10.21037/tp-21-479/rc).

\section{Methods}

\section{Study design and participants}

All data analyzed in the current study came from two cross-sectional studies, respectively. Five hundred and ten participants aged 3-6 years were obtained from the project from January 2016 to the end of 2018, by typical sampling, recruited all children in kindergartens (four in total) in Chaoyang District, Beijing. After excluded children who missed anthropometric and biochemical data, 382 children were included in the present study. The other part of participants of this study was collected from the study between 2014 and 2016, by a stratified cluster sampling method, one elementary school, one junior high school and one high school were selected in Chaoyang District, Beijing, and a total of 1,207 participants aged 6-17 years were recruited. After removed missing data on anthropometric and biochemical indicators, 1,205 cases were finally included in this study. Therefore, a total of 1,587 participants aged 3-17 were included in current study.

The study was conducted in accordance with the Declaration of Helsinki (as revised in 2013). This study has been approved by the Institutional Review Board and Ethics Committee of Capital Institute of Pediatrics, China (No. SHERLL 2015031). All participants and/or their parents or guardians provided written informed consent before participation.

\section{Measurements}

Anthropometric measurements include body weight, height, WC, systolic blood pressure (SBP), diastolic blood pressure (DBP). They were collected by trained staff following standard procedures. Body weight, height and WC were collected following technical stand for physical examination for students (GB/T 26343-2010).

Body height and weight were measured by RGZ-120 without shoes and in light clothing after overnight fasting. Standing height was to the nearest $0.1 \mathrm{~cm}$, and body weight was measured to the nearest $0.1 \mathrm{~kg}$. WC was measured to the nearest $0.1 \mathrm{~cm}$ at a point midway between the lowest rib and the iliac crest in a horizontal plane using non-elastic tape. All students' height, weight and WC were measured 
twice. BMI was calculated as weight $(\mathrm{kg})$ divided by the square of the height $(\mathrm{m}), \mathrm{WHtR}$ was calculated as $\mathrm{WC}(\mathrm{cm})$ divided by the height $(\mathrm{cm})$, ABSI was calculated according to the following formula (13):

$$
A B S I=\frac{W C}{\left[\left(B M I^{2 / 3}\right) \times\left(\text { Height }^{1 / 2}\right)\right]}
$$

ABSI-adolescents was calculated according to the following formula (20):

$$
A B S I-\text { adolescents }=\frac{W C}{\left[\left(B M I^{0.45}\right) \times\left(\text { Height }^{0.55}\right)\right]}
$$

BRI was calculated as follows (14):

$$
B R I=364.2-365.5 \times \sqrt{1-\frac{\left(\frac{W C}{2 \pi}\right)^{2}}{(0.5 \times \text { Height })^{2}}}
$$

WC and height were expressed in meters.

SBP and DBP were measured on the right upper arm in sitting position with appropriate cuff size by Omron HBP1100 electronic blood pressure monitor, all the subjects were asked sit quietly for at least 5 minutes before the measurement. Repeat the measurement 3 times and the mean of 3 measurements was used in analysis.

A fasting blood sample was collected by trained examiners after $12 \mathrm{~h}$ overnight fast. Serum levels of total cholesterol (TC), triglyceride (TG), high-density lipoprotein cholesterol (HDL-C), low-density lipoprotein cholesterol (LDL-C) and fasting plasma glucose (FPG) were measured by the Capital Institute of Pediatrics Laboratory (CNAS ISO-15189) with strict quality control.

\section{Definitions of outcomes}

Hypertension, hyperglycemia, dyslipidemia and abdominal obesity are considered as CMRFs (23). Hypertension was defined as SBP and/or DBP $\geq$ the $95^{\text {th }}$ percentile of blood pressure of the same sex and age (24). Hyperglycemia was defined as FPG $\geq 5.6 \mathrm{mmol} / \mathrm{L}$ (25). Abnormalities in any of the following indicators were defined as dyslipidemia (26): (I) $\mathrm{TC} \geq 5.2 \mathrm{mmol} / \mathrm{L}$; (II) in 0-9 age group, TG $\geq 1.1 \mathrm{mmol} / \mathrm{L}$, in $10-18$ age group, $\mathrm{TG} \geq 1.5 \mathrm{mmol} / \mathrm{L}$; (III) LDL-C $\geq 3.4 \mathrm{mmol} / \mathrm{L}$; (IV) HDL-C $\leq 1.0 \mathrm{mmol} / \mathrm{L}$. Abdominal obesity was defined as $\mathrm{WC} \geq$ the $90^{\text {th }}$ percentile of $\mathrm{WC}$ of the same sex and age $(27,28)$. The study major outcome, clustered CMRFs, are defined as meeting at least three of the above four items.

\section{Statistical analysis}

The general characteristics of the population were analyzed according to age groups. Normally distributed variables were expressed by means and standard deviations (SDs), abnormally distributed variables were described by median and the $25^{\text {th }}$ percentile $(\mathrm{Q} 1)$ to the $75^{\text {th }}$ percentiles $(\mathrm{Q} 3)$, categorical variables were described by frequencies with proportions. Baseline characteristics between groups were compared by independent sample $t$-test or Mann-Whitney $\mathrm{U}$ test for continuous variables and the chi-squared test for categorical variables. Partial correlation analysis was used to evaluate the relationship among anthropometric indicators, and the relationship among anthropometric indicators and SBP, DBP, biochemical indicators. All the statistical analysis mentioned above were accomplished by IBM SPSS Statistics (version 25.0) and R (version 3.5.0).

ROC analysis was used to evaluate the predict ability of different anthropometric indicators to identify CMRFs. Area under the receiver operating characteristic curves (AUCs) and 95\% confidence interval (CI) were reported to demonstrate the predictive ability of indicators more intuitively. Difference among AUCs of indicators were compared by using the algorithm suggested by DeLong et al. (29), the cut-off value, sensitivity, specificity and Youden Index were provided by ROC analysis, and the optimal cut-off value of each anthropometric indicator was based on the maximum Youden Index. All the statistical analysis mentioned above were accomplished by MedCalc (version 19.6.1).

All reported $\mathrm{P}$ values were 2 -sided and $<0.05$ were recognized as statistical significance.

\section{Results}

\section{Characteristics of study participants}

A total of 1,587 subjects aged 3-17 years were enrolled in this study, considering children and adolescents are in the growth and development stage, we divided the whole study populations into two groups, namely, 3-6 years old group as children and 7-17 years old group as adolescents, and all of the baseline descriptive characteristics including anthropometric measurements, biochemical variables and prevalence of CMRFs were shown in Table 1. The prevalence of hypertension, high TC and hyperglycemia is significantly higher in adolescent group than 3-6-year-old children $(\mathrm{P}<0.05)$. 
Table 1 Descriptive characteristics of study populations

\begin{tabular}{|c|c|c|c|}
\hline & $3-6$ years $(n=458)$ & $7-17$ years $(n=1,129)$ & $P$ value \\
\hline \multicolumn{4}{|c|}{ Anthropometric measurements } \\
\hline Height (cm) & $113.8 \pm 8.0$ & $152.7 \pm 16.2$ & $<0.001^{\star \star *}$ \\
\hline Weight (kg) & $20.4 \pm 4.8$ & $49.0 \pm 17.9$ & $<0.001^{\star \star *}$ \\
\hline $\mathrm{SBP}(\mathrm{mmHg})$ & $100.6 \pm 11.9$ & $115.8 \pm 12.6$ & 0.200 \\
\hline $\mathrm{DBP}(\mathrm{mmHg})$ & $58.2 \pm 10.5$ & $66.5 \pm 9.2$ & $<0.001^{\star \star \star}$ \\
\hline $\mathrm{BMI}\left(\mathrm{kg} / \mathrm{m}^{2}\right)$ & $15.62 \pm 2.20$ & $20.36 \pm 4.55$ & $<0.001^{\star \star \star}$ \\
\hline WHtR & $0.47 \pm 0.04$ & $0.45 \pm 0.06$ & $<0.001^{\star \star \star}$ \\
\hline $\mathrm{BRI}$ & $2.78 \pm 0.78$ & $2.51 \pm 1.15$ & $<0.001^{\star \star \star}$ \\
\hline \multicolumn{4}{|l|}{ Biochemical indicators } \\
\hline TC (mmol/L) & $4.2 \pm 0.8$ & $4.4 \pm 0.8$ & 0.491 \\
\hline $\mathrm{TG}(\mathrm{mmol} / \mathrm{L})$ & $0.7(0.6,0.9)$ & $0.7(0.6,1.0)$ & 0.577 \\
\hline HDL-C (mmol/L) & $1.5 \pm 0.3$ & $1.5 \pm 0.3$ & 0.439 \\
\hline LDL-C (mmol/L) & $2.5 \pm 0.6$ & $2.4 \pm 0.7$ & 0.160 \\
\hline FPG (mmol/L) & $4.8 \pm 0.3$ & $5.0 \pm 0.5$ & 0.084 \\
\hline \multicolumn{4}{|l|}{ CMRFs } \\
\hline High LDL-C, \% & $14(6.0)$ & $84(7.5)$ & 0.412 \\
\hline Hyperglycemia, \% & $1(0.4)$ & $97(8.7)$ & $<0.001^{\star \star \star}$ \\
\hline Abdominal obesity, \% & $123(27.1)$ & $334(29.6)$ & 0.323 \\
\hline Clustered CMRFs, \% & $6(2.6)$ & $54(4.8)$ & 0.137 \\
\hline
\end{tabular}

*, $\mathrm{P}<0.05$; ${ }^{* *}, \mathrm{P}<0.01$; ${ }^{* *}, \mathrm{P}<0.001$. WC, waist circumference; SBP, systolic blood pressure; DBP, diastolic blood pressure; BMI, body mass index; WHtR, waist-to-height ratio; ABSI, a body shape index; ABSI-adolescents, ABSI for adolescents; BRI, body roundness index; TC, total cholesterol; TG, triglyceride; HDL-C, high-density lipoprotein cholesterol; LDL-C, low-density lipoprotein cholesterol; FPG, fasting plasma glucose; CMRF, cardio-metabolic risk factor. Clustered CMRFs: in the four items of hypertension, dyslipidemia, IFG and abdominal obesity, meet three or more.

\section{Correlation test}

After adjusted age within each group, the correlation among anthropometric indicators was listed in Table 2. ABSI were negatively correlated with BMI in children $(\mathrm{r}=-0.284, \mathrm{P}<0.001)$, while in adolescents, the two were not correlated significantly. In 3-6 age group, ABSI-adolescents were positively correlated with $\mathrm{WC}(\mathrm{r}=0.727, \mathrm{P}<0.001)$, BMI $(r=0.218, \mathrm{P}<0.001)$ and WHtR $(r=0.752, \mathrm{P}<0.001)$, 
Table 2 Partial correlation coefficients among anthropometric indicators (adjusted for sex and age within each group)

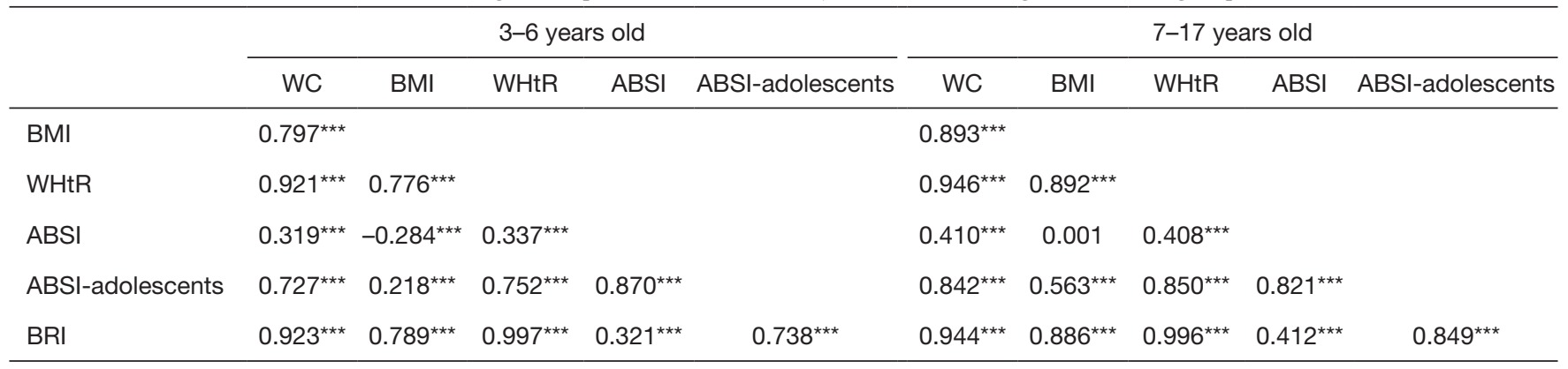

${ }^{\star \star \star}, \mathrm{P}<0.001$. WC, waist circumference; BMI, body mass index; WHtR, waist-to-height ratio; ABSI, a body shape index; ABSI-adolescents, ABSI for adolescents; BRI, body roundness index.

Table 3 Partial correlation coefficients between anthropometric indicators and biochemical indicators (adjusted for sex and age within each group)

\begin{tabular}{|c|c|c|c|c|c|c|c|c|c|c|c|c|}
\hline & \multicolumn{6}{|c|}{$3-6$ years old } & \multicolumn{6}{|c|}{$7-17$ years old } \\
\hline DBP & $0.266^{\star \star \star}$ & $0.298^{\star \star \star}$ & $0.228^{\star \star}$ & -0.087 & 0.068 & $0.240^{\star \star \star}$ & $0.108^{\star \star \star}$ & $0.119^{\star \star \star}$ & $0.122^{\star \star \star}$ & 0.018 & $0.082^{\star \star}$ & $0.127^{\star \star \star}$ \\
\hline HDL-C & -0.123 & -0.063 & -0.120 & -0.084 & -0.126 & -0.120 & $-0.381^{* \star *}$ & $-0.351^{\star \star \star}$ & $-0.354^{\star \star \star *}$ & $-0.130^{\star \star \star}$ & $-0.305^{\star \star \star}$ & $-0.345^{\star \star \star}$ \\
\hline LDL-C & $0.176^{\star \star}$ & $0.142^{*}$ & $0.224^{\star \star}$ & 0.094 & $0.179^{\star \star}$ & $0.228^{\star \star}$ & $0.253^{\star \star \star}$ & $0.241^{\star \star *}$ & $0.267^{\star \star \star}$ & $0.103^{\star \star}$ & $0.222^{\star \star \star}$ & $0.269^{\star \star \star}$ \\
\hline FPG & $0.151^{\star}$ & 0.118 & $0.133^{*}$ & 0.025 & 0.097 & 0.126 & $0.096^{\star \star}$ & 0.048 & $0.080^{\star *}$ & $0.104^{\star \star}$ & $0.115^{\star \star \star}$ & $0.079^{\star \star}$ \\
\hline
\end{tabular}

*, $\mathrm{P}<0.05$; ${ }^{* *}, \mathrm{P}<0.01 ;{ }^{* \star}, \mathrm{P}<0.001$. WC, waist circumference; BMI, body mass index; WHtR, waist-to-height ratio; ABSI, a body shape index; ABSI-adolescents, ABSI for adolescents; BRI, body roundness index; SBP, systolic blood pressure; DBP, diastolic blood pressure; TC, total cholesterol; TG, triglyceride; HDL-C, high-density lipoprotein cholesterol; LDL-C, low-density lipoprotein cholesterol; FPG, fasting plasma glucose; IFG, impaired fasting glucose.

and with age increased, the correlation coefficients increased to $0.842,0.563$ and $0.850(\mathrm{P}<0.001)$, respectively. For BRI, there were strong correlations among BRI and WC, BMI, WHtR and ABSI-adolescents in both age group $(\mathrm{P}<0.001)$.

Table 3 shows the correlation matrix between anthropometric indicators and biomarkers. In children group, both ABSI and ABSI-adolescents were not significantly correlated with biomarkers, except ABSI with SBP $(r=-0.132, \mathrm{P}<0.05)$ and ABSI-adolescents with LDL-C $(r=0.179, \mathrm{P}<0.05)$, and BRI was not related to HDL-C and FPG statistically. However, in adolescent group, ABSI, ABSI-adolescents and BRI were significantly correlated with most biomarkers, except ABSI with SBP, DBP and TC.

\section{Comparison of AUCs for different indicators}

The AUCs of anthropometric indicators for CMRFs in different age groups were listed in Table 4 and Table 5. In 3-6 years group, BMI, WHtR, ABSI-adolescents and BRI showed excellent ability to identity clustered CMRFs in both genders, while ABSI was failed to Identify high-risk children from all the participants. In 7-17 years group, WHtR and BRI showed the highest and equal AUCs in identifying hypertension ( $0.71,95 \%$ CI: $0.66-0.75$ for boys, $0.61,95 \%$ CI: $0.55-0.66$ for girls), dyslipidemia (0.65, $95 \%$ CI: $0.61-$ 0.70 for boys, $0.59,95 \%$ CI: $0.53-0.64$ for girls), abdominal obesity (0.98, 95\% CI: $0.97-0.99$ for boys, $0.98,95 \%$ CI: 0.96-0.99 for girls) and clustered CMRFs (0.85, 95\% CI: $0.81-0.88$ for boys, $0.86,95 \%$ CI: $0.83-0.89$ for girls). 


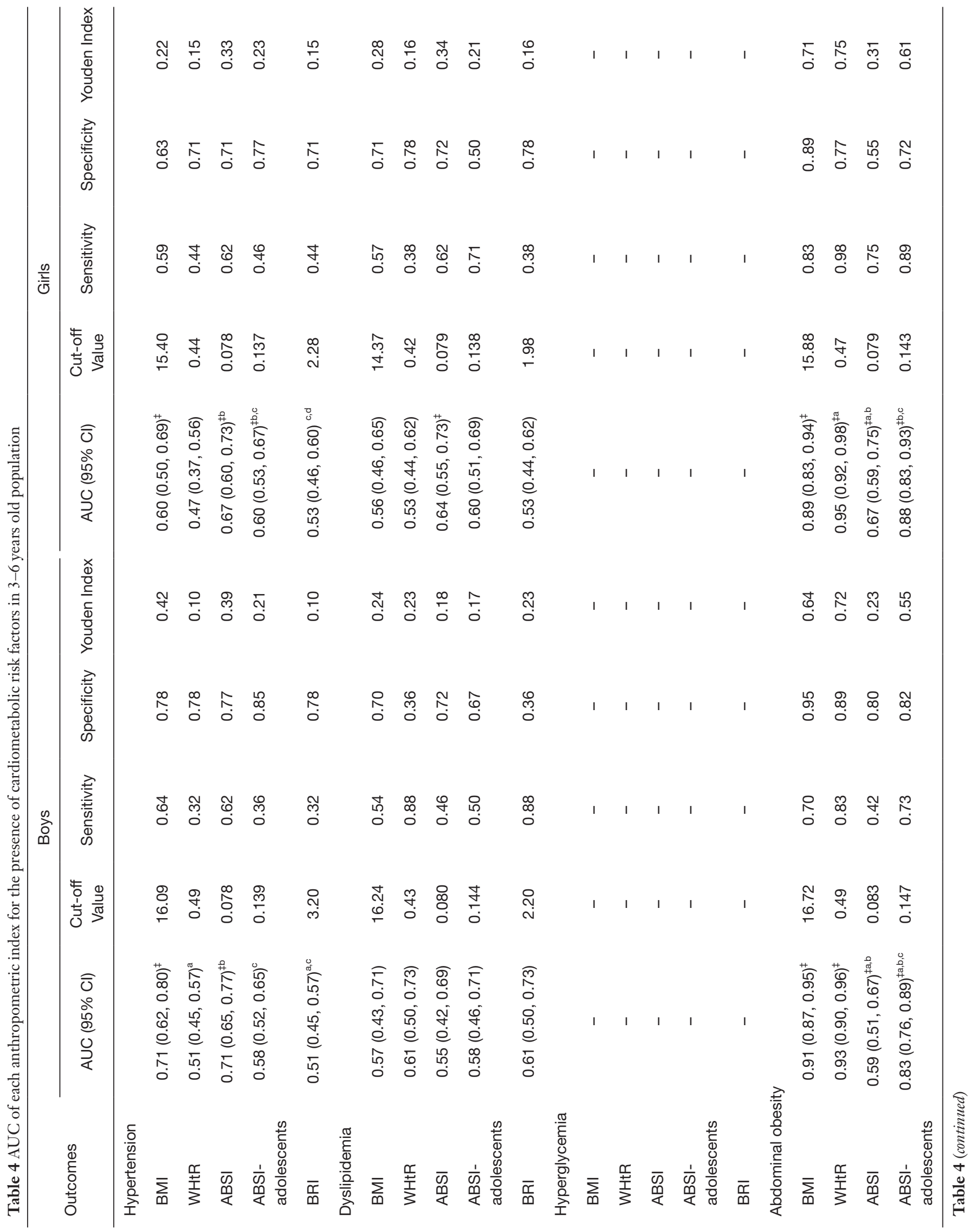




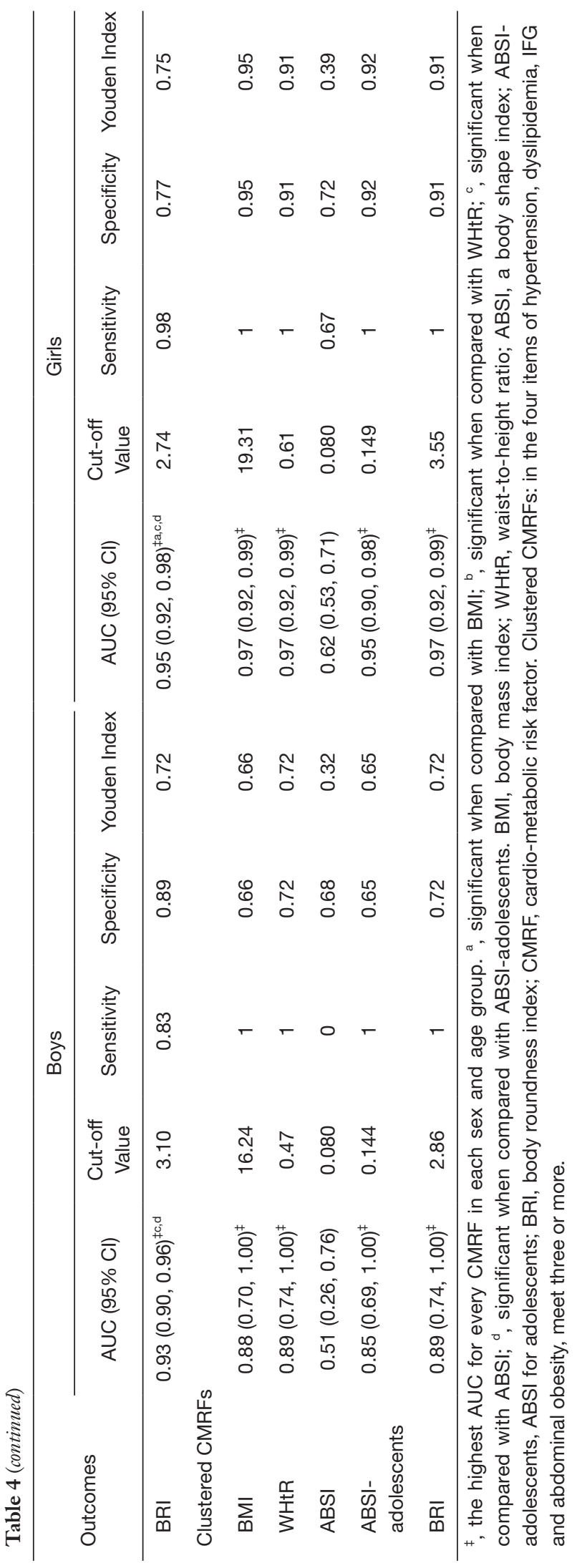

In general, no matter in which age group, for clustered CMRFs, the order of the recognition ability of the five indicators was as follows: WHtR $=\mathrm{BRI}>\mathrm{BMI}>\mathrm{ABSI}-$ adolescent $>$ ABSI, and only ABSI showed significantly difference between age groups $(\mathrm{P}<0.05$, results not shown). However, we also compared the difference between two age groups, with the increased age, the AUCs in girls were significantly different while in boys not (except ABSI). While in the same age group, there was no significant difference in the ability of each index to screen clustered CMRFs between genders ( $\mathrm{P}>0.05$, results not shown).

\section{Discussion}

In the current cross-sectional study, we compared three new anthropometric indicators (ABSI, ABSI-adolescents and $\mathrm{BRI}$ ) with BMI and WHtR for their usefulness in screening single and clustered CMRFs. Our study demonstrated that in two age groups, there is no significant difference in the ability of BMI, WHtR and BRI to recognize clustered CMRFs in both genders, BMI, WHtR, ABSIadolescents and BRI showed satisfied screening abilities, however, ABSI's performance was disappointing. For single CMRF, BMI and ABSI showed better ability to recognize hypertension in 3-6 years old children, while all the five indicators showed poor abilities to screen dyslipidemia and hyperglycemia in this age group. In 7-17 years group, compared with BMI, ABSI and ABSI-adolescents, WHtR and BRI showed significantly better and equal capability in identifying hypertension, dyslipidemia and abdominal obesity, whether in boys or girls. However, five indicators still failed in distinguishing hyperglycemia in boys, BMI and ABSI performed better in girls for hyperglycemia screening.

Briefly, our findings suggested that WHtR and BRI could be better predictors of single or clustered CMRFs in 7-17 years old population, while ABSI and ABSIadolescents were not.

Although there are a small number of studies that have evaluated the potential of ABSI and BRI in predicting CMRFs, due to the differences in ethnicity and age of the study population, there is no consensus about whether these two indicators are better than BMI. Duncan et al. (30) compared ABSI, BMI and WC's ability to predict resting blood pressure in Portuguese adolescents, and results showed that ABSI was a better predictor of BP than BMI and WC, while a research based on Chinese adolescents showed that ABSI was a weak predictor of hypertension compared with BMI, WC and WHtR (31). He et al. (32) 


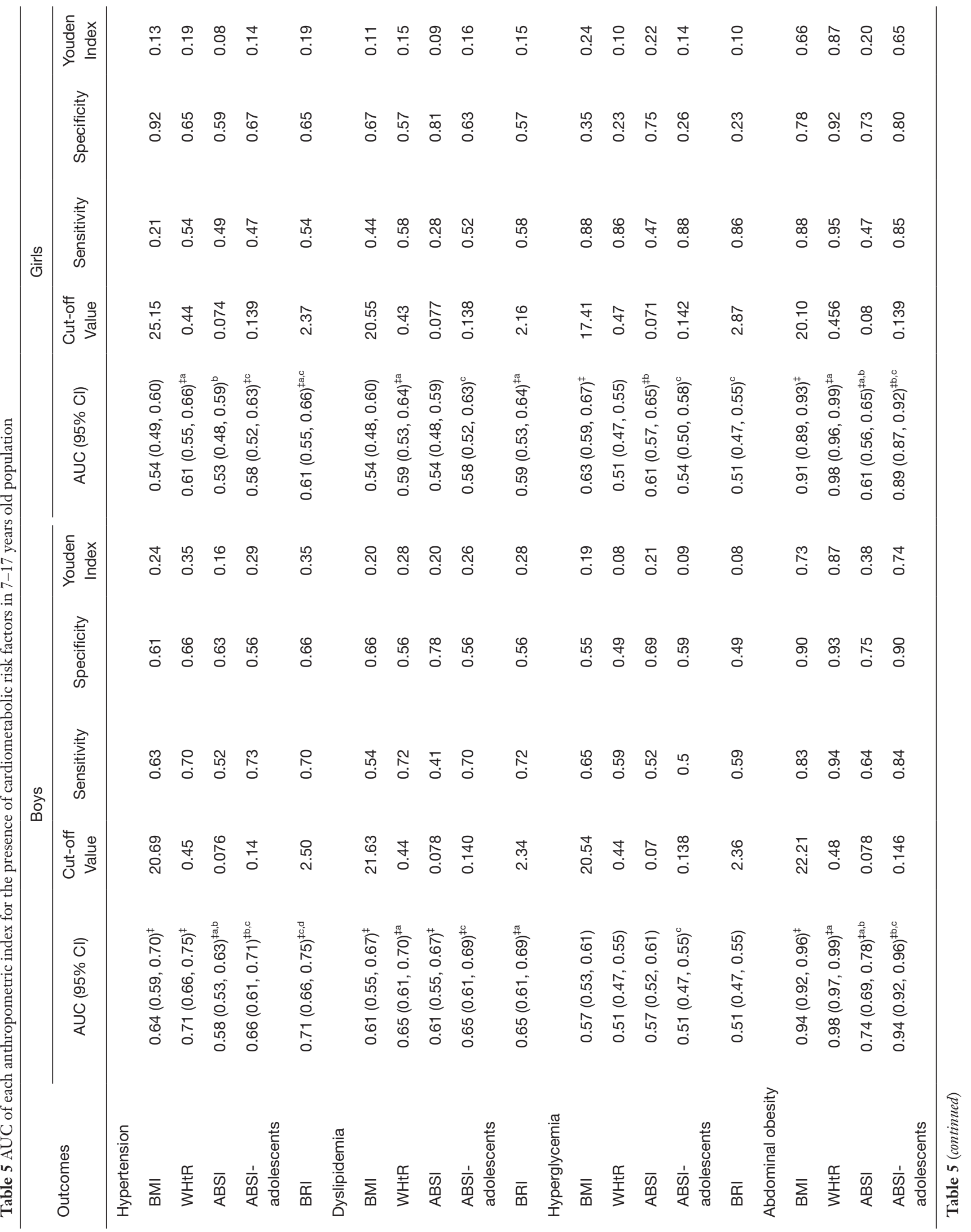




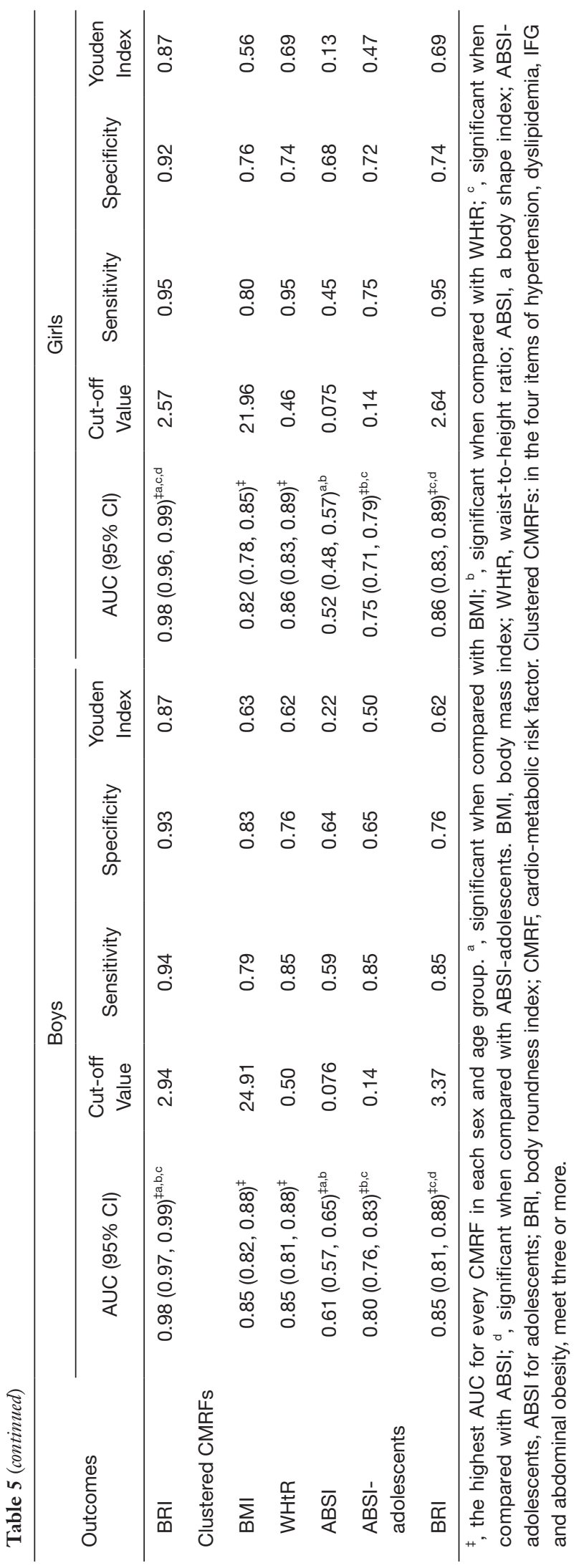

found that ABSI could be used as independent diabetes predictor in Chinese adults. Considering ABSI is related to age, $\mathrm{Xu}$ et al. (20) adjusted the scaling exponents of ABSI and suggested to use ABSI-adolescents in adolescents, in their study, ABSI-adolescents was not related to BMI, and ABSI-adolescents was not associated with BP, but was more associated with pre-diabetes than BMI. However, ABSIadolescents were positive related to $\mathrm{BMI}$ in both age groups $(\mathrm{P}<0.001)$, and connected to SBP and DBP significantly in 7-17-year-old adolescents, failed to identify hypertension and hyperglycemia for both boys and girls aged 3-17 years in our study.

BRI as another novel anthropometric measurement index developed by Thomas et al. (14) can improve predictions of $\mathrm{BF} \%$ and VAT\% slightly compared with $\mathrm{BMI}$ and WC, and provide visual results. Tian et al. (19) demonstrated that BRI showed the best capability of identify a cluster of cardiometabolic abnormalities in adults than BMI and WHtR, and ABSI was the weakest. Zhao et al. (33) also suggested that BRI is a better alternative index for assessing diabetes than ABSI and BMI. However, Maessen et al. (18) claimed that although BRI are able to determine CVD presence, the capacity of it still inferior to BMI and WC. Our results found that BRI showed good ability in identify clustered CMRFs, while it failed to recognize hyperglycemia in 7-17 age group, and could not distinguish dyslipidemia in two age group. In addition, we noticed that in correlation analysis among anthropometric indicators showed the coefficients between WHtR and BRI were 0.996-0.997 $(\mathrm{P}<0.001)$, which means these two indicators are strong correlation, the AUCs for single or clustered CMRFs of WHtR and BRI were exactly same in each age group, and the ROC curves of the two indicators were also completely overlapped, study in adults shows the same ability in screen CMRFs $(19,34,35)$. However, Chang et al. (17) found BRI performed better in predicting the presence of left ventricular hypertrophy (LVH) than BMI, WC and WHtR. All these evidence shows that BRI could provide additional information compared to BMI and WHtR, while there is relatively little evidence in children and adolescents currently, and this requires further studies pay more attention to.

The present study has several limitations. First, the cross-sectional design of the study made it difficult to establish causal relationships between anthropometric indicators and CMRFs. Second, because of there is lack of consensus about the definition of clustered CMRFs, it is difficult to compare our results with others. Third, our 
samples are all from Beijing, China, and sample size is not big enough, therefore, conclusions need to be verified in more representative research objects. However, as far as we know, this is the first study discussed the relationship among ABSI, ABSI-adolescents and BRI with CMRFs in children aged 3 to 17 years old, and we compared their identify capability with two traditional indicators (BMI and WHtR). Second, although anthropometric indicators have been proved be useful in identify individual CMRF, considering anthropometric indicators will be more efficient in screening clustered CMRFs, we compared their screening capabilities for single or clustered CMRFs separately. Thirdly, as anthropometric measurements affected by age and sex, we adjusted age and sex in partial correlation analysis, and calculated the AUCs by age group and sex, respectively.

\section{Conclusions}

In the present study, we demonstrated that BRI and WHtR were superior anthropometric indicators compared with ABSI, ABSI-adolescents and BMI in identify abdominal obesity and clustered CMRFs, and ABSI showed the worst ability in distinguishing all the single or clustered CMRFs. All the five indicators were failed to identify dyslipidemia and hyperglycemia in both children and adolescents. Considering BRI was initially developed to evaluate health status in adults, further studies could explore whether it is necessary to adjust the scaling exponents of BRI to be suited in children and adolescents.

\section{Acknowledgments}

Funding: This work was supported by The Special Fund of the Pediatric Medical Coordinated Development Center of Beijing Hospitals Authority (No. XTCX201813).

\section{Footnote}

Reporting Checklist: The authors have completed the STARD reporting checklist (available at https://tp.amegroups.com/ article/view/10.21037/tp-21-479/rc).

Data Sharing Statement: Available at https://tp.amegroups. com/article/view/10.21037/tp-21-479/dss

Peer Review File: Available at https://tp.amegroups.com/ article/view/10.21037/tp-21-479/prf
Conflicts of Interest: All authors have completed the ICMJE uniform disclosure form (available at https://tp.amegroups. com/article/view/10.21037/tp-21-479/coif). The authors have no conflicts of interest to declare.

Ethical Statement: The authors are accountable for all aspects of the work in ensuring that questions related to the accuracy or integrity of any part of the work are appropriately investigated and resolved. The study was conducted in accordance with the Declaration of Helsinki (as revised in 2013). This study has been approved by the Institutional Review Board and Ethics Committee of Capital Institute of Pediatrics, China (No. SHERLL 2015031). All participants and/or their parents or guardians provided written informed consent before participation.

Open Access Statement: This is an Open Access article distributed in accordance with the Creative Commons Attribution-NonCommercial-NoDerivs 4.0 International License (CC BY-NC-ND 4.0), which permits the noncommercial replication and distribution of the article with the strict proviso that no changes or edits are made and the original work is properly cited (including links to both the formal publication through the relevant DOI and the license). See: https://creativecommons.org/licenses/by-nc-nd/4.0/.

\section{References}

1. Saliba LJ, Maffett S. Hypertensive Heart Disease and Obesity: A Review. Heart Fail Clin 2019;15:509-17.

2. Cook S, Kavey RE. Dyslipidemia and pediatric obesity. Pediatr Clin North Am 2011;58:1363-73, ix.

3. Di Bonito P, Pacifico L, Chiesa C, et al. Impaired fasting glucose and impaired glucose tolerance in children and adolescents with overweight/obesity. J Endocrinol Invest 2017;40:409-16.

4. Seravalle G, Grassi G. Obesity and hypertension. Pharmacol Res 2017;122:1-7.

5. Juonala M, Magnussen CG, Berenson GS, et al. Childhood adiposity, adult adiposity, and cardiovascular risk factors. N Engl J Med 2011;365:1876-85.

6. Llewellyn A, Simmonds M, Owen CG, et al. Childhood obesity as a predictor of morbidity in adulthood: a systematic review and meta-analysis. Obes Rev 2016;17:56-67.

7. Giudici KV, Martini LA. Comparison between body mass index and a body shape index with adiponectin/leptin ratio and markers of glucose metabolism among adolescents. 
Ann Hum Biol 2017;44:489-94.

8. Gómez-Ambrosi J, Silva C, Galofré JC, et al. Body mass index classification misses subjects with increased cardiometabolic risk factors related to elevated adiposity. Int J Obes (Lond) 2012;36:286-94.

9. Moore SC. Waist versus weight: which matters more for mortality? Am J Clin Nutr 2009;89:1003-4.

10. Arellano-Ruiz P, García-Hermoso A, García-Prieto JC, et al. Predictive Ability of Waist Circumference and Waistto-Height Ratio for Cardiometabolic Risk Screening among Spanish Children. Nutrients 2020;12:415.

11. Lo K, Wong M, Khalechelvam P, et al. Waist-to-height ratio, body mass index and waist circumference for screening paediatric cardio-metabolic risk factors: a metaanalysis. Obes Rev 2016;17:1258-75.

12. Browning LM, Hsieh SD, Ashwell M. A systematic review of waist-to-height ratio as a screening tool for the prediction of cardiovascular disease and diabetes: 0.5 could be a suitable global boundary value. Nutr Res Rev 2010;23:247-69.

13. Krakauer NY, Krakauer JC. A new body shape index predicts mortality hazard independently of body mass index. PLoS One 2012;7:e39504.

14. Thomas DM, Bredlau C, Bosy-Westphal A, et al. Relationships between body roundness with body fat and visceral adipose tissue emerging from a new geometrical model. Obesity (Silver Spring) 2013;21:2264-71.

15. Cheung YB. "A Body Shape Index" in middle-age and older Indonesian population: scaling exponents and association with incident hypertension. PLoS One 2014;9:e85421.

16. Park Y, Kim NH, Kwon TY, et al. A novel adiposity index as an integrated predictor of cardiometabolic disease morbidity and mortality. Sci Rep 2018;8:16753.

17. Chang Y, Guo X, Li T, et al. A Body Shape Index and Body Roundness Index: Two New Body Indices to Identify left Ventricular Hypertrophy among Rural Populations in Northeast China. Heart Lung Circ 2016;25:358-64.

18. Maessen MF, Eijsvogels TM, Verheggen RJ, et al. Entering a new era of body indices: the feasibility of a body shape index and body roundness index to identify cardiovascular health status. PLoS One 2014;9:e107212.

19. Tian S, Zhang X, Xu Y, et al. Feasibility of body roundness index for identifying a clustering of cardiometabolic abnormalities compared to BMI, waist circumference and other anthropometric indices: the China Health and Nutrition Survey, 2008 to 2009. Medicine (Baltimore) 2016;95:e4642.
20. Xu Y, Yan W, Cheung YB. Body shape indices and cardiometabolic risk in adolescents. Ann Hum Biol 2015;42:70-5.

21. Mameli C, Krakauer NY, Krakauer JC, et al. The association between a body shape index and cardiovascular risk in overweight and obese children and adolescents. PLoS One 2018;13:e0190426.

22. Ismail NA, Ragab SH, El Baky AMNEDA, et al. Potential Role of New Anthropometric Parameters in Childhood Obesity with or Without Metabolic Syndrome. Open Access Maced J Med Sci 2019;7:3930-6.

23. Dou Y, Jiang Y, Yan Y, et al. Waist-to-height ratio as a screening tool for cardiometabolic risk in children and adolescents: a nationwide cross-sectional study in China. BMJ Open 2020;10:e037040.

24. Fan H, Yan Y, Mi J, et al. Updating blood pressure references for Chinese children aged 3-17 years. Chinese Journal of Hypertension 2017;25:428-35.

25. American Diabetes Association. Diagnosis and classification of diabetes mellitus. Diabetes Care 2014;37 Suppl 1:S81-90.

26. Expert Panel on Integrated Guidelines for Cardiovascular Health and Risk Reduction in Children and Adolescents, National Heart, Lung, and Blood Institute. Expert Panel on Integrated Guidelines for Cardiovascular Health and Risk Reduction in Children and Adolescents. Pediatrics 2011;128:S213-56.

27. Zong XN, Li H, Zhang YQ, et al. Percentile reference value of waist circumference for Chinese children aged 3-7 years. Zhonghua Liu Xing Bing Xue Za Zhi 2020;41:1286-90.

28. Ma GS, Ji CY, Ma J, et al. Waist circumference reference values for screening cardiovascular risk factors in Chinese children and adolescents aged $7-18$ years. Zhonghua Liu Xing Bing Xue Za Zhi 2010;31:609-15.

29. DeLong ER, DeLong DM, Clarke-Pearson DL. Comparing the areas under two or more correlated receiver operating characteristic curves: a nonparametric approach. Biometrics 1988;44:837-45.

30. Duncan MJ, Mota J, Vale S, et al. Associations between body mass index, waist circumference and body shape index with resting blood pressure in Portuguese adolescents. Ann Hum Biol 2013;40:163-7.

31. Tong Y, Han E. Associations between body shape, body adiposity and other indices: a case study of hypertension in Chinese children and adolescents. Ann Hum Biol 2019;46:460-6.

32. He S, Chen X. Could the new body shape index predict 
the new onset of diabetes mellitus in the Chinese population? PLoS One 2013;8:e50573.

33. Zhao Q, Zhang K, Li Y, et al. Capacity of a body shape index and body roundness index to identify diabetes mellitus in Han Chinese people in Northeast China: a cross-sectional study. Diabet Med 2018;35:1580-7.

34. Xu J, Zhang L, Wu Q, et al. Body roundness index

Cite this article as: Chen R, Ji L, Chen Y, Meng L. Weightto-height ratio and body roundness index are superior indicators to assess cardio-metabolic risks in Chinese children and adolescents: compared with body mass index and a body shape index. Transl Pediatr 2022;11(3):318-329. doi: 10.21037/ tp-21-479 is a superior indicator to associate with the cardiometabolic risk: evidence from a cross-sectional study with 17,000 Eastern-China adults. BMC Cardiovasc Disord 2021;21:97.

35. Feng J, He S, Chen X. Body Adiposity Index and Body Roundness Index in Identifying Insulin Resistance Among Adults Without Diabetes. Am J Med Sci 2019;357:116-23. 